conservative and asymmetric synthesis of many progeny plus strands. The first model is highly unorthodox because it requires the replication of a nucleic acid molecule by some other mechanism than WatsonCrick base pairing. The second model predicts the existence in an infected cell of a heterogencous population of viral RNA molecules: single stranded progeny molecules, pure double stranded molecules, a replicative form, and double stranded molecules with nascent single stranded molecules attached, a replicative intermediate.

Several workers from 1964 onwards reported the presence of replicative form and replicative intermediate in phage infected Escherichia coli. Controversy suddenly arose when Haruna and Spicgelman (1966), having achieved the isolation of $Q \beta$ RNA replicase which was stable and showed template specificity in vitro, were unable to find ovidence of a complementary minus strand during the in vitro replication of Q $\beta$ RNA. Weissman and Feix (1966), on the other hand, with $Q \beta$ replicase donated by Spiegelman, demonstrated with hybridization techniques the existence of such minus strands. At the time, Spiegelman did not accept these results, but subsequent work done in his laboratory has restored unity to the field. Mills, Pace and Spiegelman (Proc. Nat. Acad. Sci, 56, $1778 ; 1966)$ showed the existence of a double stranded Q $\beta$ RNA molecule, and Bishop, Claybrook, Pace and Spiegelman (Proc. Nat. Acad. Sci., 5\%, 1474; 1967) with polyacrylamide gel electrophoresis have isolated $\mathrm{Q} \beta \mathrm{RNA}$ complexes with properties similar to replicative form and replicative intermediate described by Francke and Hofschncider (1966), Franklin (1966) and Erikson et al. (1967). Spiegelman et al. are now trying to show that these double stranded molecules contain complete minus and plus strands. They caution that although their latest results are consistent with the second model for phage RNA replication they do not unequivocally establish it. However, all these data with those of Feix, Slor and Weissman (Proc. Nat. Acad. Sci., 5\%, 1401; 1967), who present evidence for the synthesis of complete $Q \beta$ minus strands during in vitro replication, make it increasingly difficult to believe that phage RNA is replicated without the synthesis of a complementary minus strand.

Granted the synthesis of a minus strand, there remains the problem of whether or not it acts as a template for synthesis of progeny strands when part of a double helical molecule with a parental plus strand. Feix et al. find most of the minus strands made are not part of a double helical molecule but are found in a complex sedimenting at about $40 s$ which probably consists of a minus and plus strand which are not hydrogen bonded, and a replicase molecule. Thoy suggest that in vitro and probably in vivo the template for progeny strand synthesis is a single minus strand, not a double helical molecule. This is supported by the finding that before denaturation the replicative form and replicative intermediate RNA do not serve in vitro as a template for $Q \beta$ replicase. Since the RNA molecules in the $40 s$ complex can be converted to a double stranded form by phenol, they believe that the replicative form and replicative intermediate normally isolated from phage infected cells by phenol extraction are derived from the in vivo replicating complex but are no longer in the native state. Further experiments may be expected to test these ideas.

\section{lonizing Groups in Proteins}

\section{from our Correspondent in Molecular Biology}

THE biological activity of proteins and the transformations which they will undergo are in many cases known to be governed by particular ionizing groups. That such groups are frequently grossly abnormal in effective $p \mathrm{~K}$ in the native protein is now a commonplace, and it is also known that they are in general restored to normal when the protein is denatured and that they may change in response to some external event, such as the arrival of a substrate or effector molecule. Considerable interest therefore attaches to the identification of individual ionizing groups and the study of their behaviour in isolation. A method of observing only the $\alpha$-amino group, first applied by Hirs and his collaborators in work on ribonuclease, has now been used to good purpose on haemoglobin by Hill and Davies (J. Biol. Chem., 242, 2005; 1967).

The principle of the method lies in the well-established fact that fluorodinitrobenzene (FDNB), the reagent originally introduced by Sanger for the determination of end-groups, reacts with amino groups only in their uncharged (high $p \mathrm{H}$ ) state. Thus the greater the degree of protonation of the amino groups, the slower the reaction. If now the reaction rate is followed as a function of $p \mathbf{H}$, a measure of the ionization state of the amino group in question will in effect be obtained at each $p \mathrm{H}$ so that the effective $p \mathrm{~K}$ can be determined. The rate of the reaction with, in this case, the $\alpha$-amino groups of the haemoglobin $\alpha$-chains is followed by hydrolysis and estimation of reacted groups in a stand. ard manner. Hill and Davies have shown that when the dipeptide valylleucine (which is the N-terminal sequence in the haemoglobin chain) is subjected to this method, the $p \mathrm{~K}$ of the $\alpha$-amino group agrees closely with the value 7.9 from potentiometric titration. In native haemoglobin, on the other hand, the $p \mathrm{~K}$ of the $\alpha$-amino groups is $6 \cdot 7$, more than one $p \mathrm{H}$ unit lower.

Apart from demonstrating the effectiveness of the method, this result is of interest in itself, for a recent analysis by 'Tanford and Nozaki of the titration curve of human haemoglobin led to the conclusion that the $\alpha$-amino groups do not titrate in the expected range. On the basis of the crystallographic model, Perutz has also suggested that these same amino groups are paired with the positively charged guanidinium groups of the C-terminal arginines in the partnering $\alpha$-chains, which should account for the anomalous $p \mathrm{~K} s$.

Another article which concerns itself with the observation of a single class of ionizing groups comes from Vijai and Foster (Biochemistry, 6, 1152; 1967). They have established the nature of the "masked" ionizing groups in bovine serum albumin, which become available for titration when the protein undergoes a structural change at acid $p \mathrm{H}$ - the groups turn out to be carboxylate groups and they were identified by infrared spectroscopy. By using difference spectra it is possible to follow quantitatively the disappearance of a frequency of the carboxylate group as the $p \mathrm{H}$ is lowered. By comparison with the potentiometric titration curve, it is shown that in the acid region only carboxylate groups are being titrated, and that it is such groups which are exposed when the acid conformational transition occurs. With some refinements, it has been shown that about 40 of the 100 carboxylate groups present are inaccessible in the native protein. 